\title{
Emergence of statistically validated financial intraday lead-lag relationships
}

\author{
CHESTER CURME†, MICHELE TUMMINELLO $\$$, ROSARIO N. MANTEGNA§I, \\ H. EUGENE STANLEY $\dagger$ and DROR Y. KENETT*†
}

\author{
$\dagger$ Center for Polymer Studies and Department of Physics, Boston University, Boston, MA, USA \\ $\$$ Dipartimento di Scienze Economiche, Aziendali e Statistiche, University of Palermo, Viale delle Scienze, Ed. 13, Palermo \\ IT-90128, Italy \\ §Center for Network Science and Department of Economics, Central European University, Nador utca 9, 1051 Budapest, \\ Hungary \\ IIDipartimanto di Fisica e Chimica, University of Palermo, Viale delle Scienze, Ed. 18, Palermo IT-90128, Italy
}

(Received 21 December 2013; accepted 9 April 2014)

\begin{abstract}
According to the leading models in modern finance, the presence of intraday lead-lag relationships between financial assets is negligible in efficient markets. With the advance of technology, however, markets have become more sophisticated. To determine whether this has resulted in an improved market efficiency, we investigate whether statistically significant lagged correlation relationships exist in financial markets. We introduce a numerical method to statistically validate links in correlationbased networks, and employ our method to study lagged correlation networks of equity returns in financial markets. Crucially, our statistical validation of lead-lag relationships accounts for multiple hypothesis testing over all stock pairs. In an analysis of intraday transaction data from the periods 2002-2003 and 2011-2012, we find a striking growth in the networks as we increase the frequency with which we sample returns. We compute how the number of validated links and the magnitude of correlations change with increasing sampling frequency, and compare the results between the two data-sets. Finally, we compare topological properties of the directed correlation-based networks from the two periods using the in-degree and out-degree distributions and an analysis of three-node motifs. Our analysis suggests a growth in both the efficiency and instability of financial markets over the past decade.
\end{abstract}

Keywords: Financial markets; Stock returns; High-frequency data; Lead-lag correlations; Bootstrap method; Statistically validated networks

JEL Classification: C55, C15, G41

\section{Introduction}

Modern financial markets have developed lives of their own. This fact makes it necessary that we not only monitor financial markets as an 'auxiliary system' of the economy, but that we develop a methodology for evaluating them, their feedback on the real economy and their effect on society as a whole (Hall 2010, Cecchetti and Kharroubi 2012). The events of the recent past have clearly demonstrated that the everyday life of the majority of the world's population is tied to the well-being of the financial system. Individuals are invested in stock markets either directly or indirectly, and shocks to the system (be they endogenous or exogenous) have an immense and immediate impact. Thus, the need for a robust and efficient

*Corresponding author. Email: drorkenett@gmail.com All authors contributed equally to this manuscript. financial system is becoming stronger and stronger. These two critical concepts have been discussed and heatedly debated for the past century, with the efficient market hypothesis (EMH) in the center of the debate.

The EMH (Tobin 1969, Malkiel and Fama 1970) stipulates that all available information (or only past prices in the weak variant of the hypothesis) is already reflected in the current price and it is therefore not possible to predict future values in any statistical method based on past records (Malkiel 2003). The EMH has been questioned by applying statistical tests to New York Stock Exchange (NYSE) returns (Lo and MacKinlay 1988, Shmilovici et al. 2003) in which the authors formulated the problem equivalent to the EMH, and showed by contrast that an efficient compression algorithm they proposed was able to utilize structure in the data-which would not be possible if the hypothesis were in fact true. The possibility for such 
compression suggests the data must be somehow structured. This encourages us to explore methods of modelling and exploring this structure in ways that can be applied to real-world markets.

Many efforts have thus been devoted to uncovering the true nature of the underlying structure of financial markets. Much attention has been given to understanding correlations in financial markets and their dynamics, for both daily (Mantegna 1999, Gopikrishnan et al. 2000, Cizeau et al. 2001, Forbes and Rigobon 2002, Campbell et al. 2008, Podobnik and Stanley 2008, Carbone 2009, Aste et al. 2010, Pollet and Wilson 2010, Kenett, Preis et al. 2012, Kenett, Raddant et al. 2012) and intra-day time scales (Bonanno et al. 2001, Borghesi et al. 2007, Tumminello, Di Matteo et al. 2007, Munnix et al. 2010). More recently, other measures of similarity have been introduced, such as Granger-causality analysis (Billio et al. 2012) and partial correlation analysis (Kenett et al. 2010), both of which aim to quantify how the behaviour of one financial asset provides information about the behaviour of a second asset. For these different measures of co-movement in financial markets, however, the main question that remains is how to uncover underlying meaningful information.

An analysis of synchronous correlations of equity returns has shown that a financial market usually displays a nested structure in which all the stock returns are driven by a common factor, e.g. a market index, and are then organized in groups of like economic activity—such as technology, services, utilities or energy - that exhibit higher values of average pair correlation. Within each group, stocks belonging to the same sub-sector of economic activity, e.g. 'insurance' and 'regional banks' within the financial sector, show an even higher correlation degree. Such a structure has been recognized using very different methods of analysis, ranging from random matrix theory (Laloux et al. 2000, Gopikrishnan et al. 2001), to hierarchical clustering (Mantegna 1999), to correlation-based networks (Mantegna 1999, Bonanno et al. 2003, Onnela et al. 2003). The several methods devised to construct correlationbased networks can be grouped into two main categories: threshold methods and topological/hierarchical methods. Both approaches start from a sample correlation matrix or, more generally, a sample similarity measure. Using the threshold method, we set a correlation threshold and construct a network in which any two nodes are linked if their correlation is larger than the threshold. As we lower the threshold value, we see the formation of groups of stocks (economic sub-sectors) that progressively merge to form larger groups (economic sectors) and finally merge into a single group (the market). The advantage of this approach is that, due to the finite length of data series, threshold networks are very robust to correlation uncertainty. The disadvantage of threshold-based networks is that it is difficult to find a single threshold value to display, in a single network, the nested structure of the correlation matrix of stock returns (see Kenett et al. 2010). Topological methods to construct correlation-based networks, such as the minimal spanning tree (MST) (Mantegna 1999, Bonanno et al. 2001, Bonanno et al. 2003, Onnela et al. 2003) or the planar Maximally filtered graph (PMFG) (Tumminello et al. 2005), are based solely on the ranking of empirical correlations. The advantage of this approach is that these methods are intrinsically hierarchical and are able to display the nested struc- ture of stock-return correlations in a financial market. The disadvantage of this approach is that these methods are less stable than threshold methods with respect to the statistical uncertainty of data series, and it is difficult to include information about the statistical significance of correlations and their ranking (Tumminello, Coronnello et al. 2007). Thus, it is a challenge of modern network science to uncover the significant relationships (links) between the components (nodes) of the investigated system (Havlin et al. 2012).

Although much attention has been devoted to the study of synchronous correlation networks of equity returns (see Tumminello et al. 2010 for a review of the topic), comparatively few results have been obtained for networks of lagged correlations (Huth and Abergel 2014). Neither method of constructing correlation-based networks is readily extendable to the study of directed lagged correlations in a financial market. The lagged correlations in stock returns are small, even at time horizons as short as $5 \mathrm{~min}$, and are thus strongly influenced by the statistical uncertainty of the estimation process. The use of topological methods to construct a lagged correlation-based network of stock returns is difficult because they only take into consideration the ranking of correlations and not their actual values. The result could be a network in which many links are simply caused by statistical fluctuations. On the other hand, standard threshold methods are also difficult to apply because it is difficult to find an appropriate threshold level and, more importantly, the threshold selected in these methods is usually the same for all stock pairs. This is a problem if we want to study lagged correlations because the statistical significance of a lagged-correlation may depend on the return distribution of the corresponding pair of stocks, and such distributions might vary across stocks - a consequence, for example, of the different liquidity of stocks.

Here, we introduce a method for filtering a lagged correlation matrix into a network of statistically validated directed links that takes into account the heterogeneity of stock return distributions. This is done by associating a $p$-value with each observed lagged correlation and then setting a threshold on $p$ values, i.e. setting a level of statistical significance corrected for multiple hypothesis testing. We apply our method to describe the structure of lagged relationships between intraday equity returns sampled at high frequencies in financial markets. In particular, we investigate how the structure of the network changes with increasing return sampling frequency, and compare the results using data from both the periods 2002-2003 and 20112012. It should be noted that the two investigated time periods are quite different if we consider that the fraction of volume exchanged by algorithmic trading in the US equity markets has increased from approximately $20 \%$ in 2003 to more than $50 \%$ in 2011. In both periods, we find a large growth in the connectedness of the networks as we increase the sampling frequency.

The paper is organized as follows. Section 2 introduces the method used to filter and validate statistically significant lagged correlations from transaction data. Section 3 analyzes the structure of the resulting networks and investigates how this structure evolves with changing return sampling frequency. In section 4, we discuss the application of our method to the construction of synchronous correlation networks. Finally, in section 5, we discuss the implications of our results for the efficiency and stability of financial markets. 


\section{Statistically validated lagged correlation networks}

We begin the analysis by calculating the matrix of logarithmic returns over given intraday time horizons. We denote by $p_{n}(t)$ the most recent transaction price for stock $n$ occurring on or before time $t$ during the trading day. We define the opening price of the stock to be the price of its first transaction of the trading day. Let $h$ be the time horizon. Then for each stock we sample the logarithmic returns,

$$
r_{n, t} \equiv \log \left(p_{n}(t)\right)-\log \left(p_{n}(t-h)\right),
$$

every $h$ minutes throughout the trading day, and assemble these time series as columns in a matrix $R$. We then filter $R$ into two matrices, $A$ and $B$, in which we exclude returns during the last period $h$ of each trading day from $A$ and returns during the first period $h$ of each trading day from $B$. From these data, we construct an empirical lagged correlation matrix $C$ using the Pearson correlation coefficient of columns of $A$ and $B$,

$$
C_{m, n}=\frac{1}{T-1} \sum_{i=1}^{T} \frac{\left(A_{m, i}-\left\langle A_{m}\right\rangle\right)\left(B_{n, i}-\left\langle B_{n}\right\rangle\right)}{\sigma_{m} \sigma_{n}},
$$

where $\left\langle A_{m}\right\rangle$ and $\sigma_{m}$ are the mean and sample standard deviation, respectively, of column $m$ of $A$, and $T$ is the number of rows in $A$ (and $B$ ). Here, we set the lag to be one time horizon $h$. A schematic of this sum is diagrammed in figure 1 .

The matrix $C$ can be considered a weighted adjacency matrix for a fully connected, directed graph. To filter the links in this graph according to a threshold of statistical significance, we apply a shuffling technique (Efron and Tibshirani 1993). The rows of $A$ are shuffled repeatedly without replacement in order to create a large number of surrogated time series of returns. After each shuffling, we re-calculate the lagged correlation matrix (2) and compare this shuffled lagged correlation matrix $\widetilde{C}$ to the empirical matrix $C$. For each shuffling, we thus have an independent realization of $\widetilde{C}$. We then construct the matrices $\underset{\sim}{U}$ and $D$, where $U_{m, n}$ is the number of realizations for which $\widetilde{C}_{m, n} \geq C_{m, n}$, and $D_{m, n}$ is the number of realizations for which $\widetilde{C}_{m, n} \leq C_{m, n}$.

From matrix $U$, we associate a one-tailed $p$-value with all positive correlations as the probability of observing a correlation that is equal to or higher than the empirically measured correlation. Similarly, from $D$, we associate a one-tailed $p$ value with all negative correlations. In this analysis, we set the threshold at $p=0.01$. We must adjust our statistical threshold, however, to account for multiple comparisons. We use the conservative Bonferroni correction for a given sample size of $N$ stocks. For example, for $N=100$ stocks the corrected threshold will be $0.01 / N^{2}=10^{-6}$. We thus construct $10^{6}$ independently shuffled surrogate time series. If $U_{m, n}=0$ we can associate a statistically-validated positive link from stock $m$ to stock $n$ ( $p=0.01$, Bonferroni correction). Likewise, if $D_{m, n}=0$ we can associate a statistically validated negative link from stock $m$ to stock $n$. In this way, we construct the Bonferroni network (Tumminello et al. 2011). In appendix 1, we discuss the probability that using our approximated method we will wrongly identify a link as statistically significant (i.e. have a false positive).

For the sake of comparison, for each time horizon $h$ we also construct the network using $p$-values corrected according to the false discovery rate (FDR) protocol (Benjamini and
Hochberg 1995). This correction is less conservative than the Bonferroni correction and is constructed as follows. The $p$-values from each individual test are arranged in increasing order $\left(p_{1}<p_{2}<\cdots<p_{N^{2}}\right)$, and the threshold is defined as the largest $k$ such that $p_{k}<k 0.01 / N^{2}$. In the FDR network, our threshold for the matrices $U$ or $D$ is thus not zero but the largest integer $k$ such that $U$ or $D$ has exactly $k$ entries fewer than or equal to $k$. From this threshold, we can filter the links in $C$ to construct the FDR network (Tumminello et al. 2011). We note that the Bonferroni network is a subgraph of the FDR network.

Because we make no assumptions about the return distributions, this randomization approach is especially useful in high-dimensional systems in which it can be difficult to infer the joint probability distribution from the data (Tumminello, Coronnello et al. 2007). We also impose no topological constraints on the Bonferroni or FDR networks. This method serves to identify the significant positive and negative lagged correlation coefficients in a way that accounts for heterogeneities in relationships between the returns of stocks. An alternative, but closely related approach, would be to construct a theoretical distribution for correlation coefficients under the null hypothesis of uncorrelated returns sampled from a given joint distribution (Biroli et al. 2007). For a desired confidence level, one could then construct a threshold correlation, beyond which empirical correlations are validated. Such an approach typically assumes equal marginal distributions for returns, and must fix a uniform correlation threshold for all relationships. At the expense of computational time, our method is flexible in that it permits heterogeneities in marginal distributions. We compare the results of the two approaches in appendix 2 .

\section{Lagged correlation networks in NYSE}

We study and compare two different data-sets. The first Dataset comprises returns of 100 companies with the largest market capitalization on the NYSE during the period 2002-2003 (501 trading days), which was investigated in Tumminello, Di Matteo et al. (2007). For the second data-set, we consider returns during the period 2011-2012 (502 trading days) of 100 companies with the largest market capitalization on the NYSE as of 31 December 2012 (retrieved from the Trades and Quotes database, Wharton Research Data Services, http://wrds-web. wharton.upenn.edu/wrds/). Market capitalization figures were obtained from Yahoo Finance web service (http://finance.yahoo. com). For each company we obtain intraday transaction records. These records provide transaction price data at a time resolution of one second. The stocks under consideration are quite liquid, helping to control for the problem of asynchronous transactions and artificial lead-lag relationships due to different transaction frequencies (De Jong et al. 1996). Transaction data were cleaned for the cancelled trades and trades reported out of sequence. We then sample returns at time horizons of 5, 15, 30,65 and $130 \mathrm{~min}$.

We report summary statistics in table 1, including the lengths of time series $T$ from equation (2), as well as the mean $\langle\rho\rangle$ and standard deviation $\sigma_{\rho}$ of synchronous Pearson correlation coefficients between distinct columns of the returns matrix $R$ for each time horizon $h$. We also show the mean $\left\langle C_{m, n}\right\rangle$ 


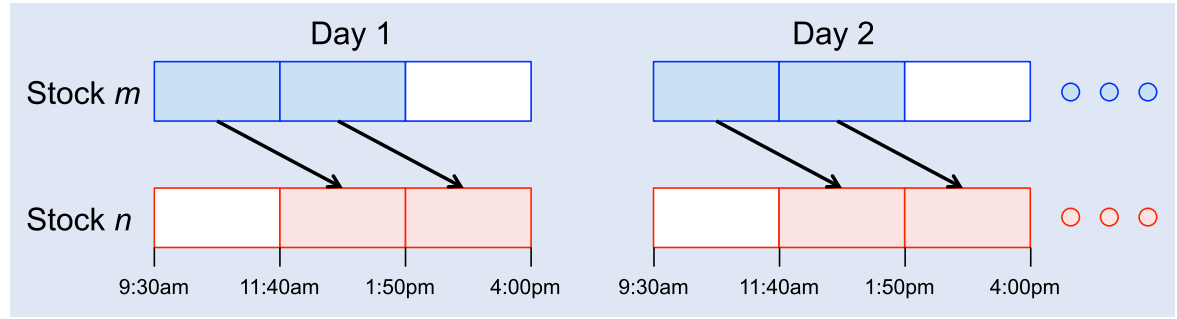

Figure 1. Schematic of lagged correlation calculation for a time horizon $h=130 \mathrm{~min}$. The sum $C_{m, n}$ is generated using products of returns from stocks $m$ and $n$ that are linked by an arrow. We consider only time horizons $h$ that divide evenly into the 390 min trading day.

Table 1. Summary statistics of 2002-2003 and 2011-2012 data-sets.

\begin{tabular}{lcccccc}
\hline Period & $T$ & $h$ & $\langle\rho\rangle$ & $\sigma_{\rho}$ & $\left\langle C_{m, n}\right\rangle$ & $\sigma_{C}$ \\
\hline \multirow{2}{*}{$2002-2003$} & 38577 & $5 \mathrm{~min}$ & 0.267 & 0.077 & 0.008 & 0.024 \\
& 12525 & $15 \mathrm{~min}$ & 0.290 & 0.092 & 0.007 & 0.025 \\
& 6012 & $30 \mathrm{~min}$ & 0.307 & 0.102 & 0.005 & 0.025 \\
& 2505 & $65 \mathrm{~min}$ & 0.317 & 0.110 & 0.015 & 0.029 \\
& 1002 & $130 \mathrm{~min}$ & 0.327 & 0.115 & 0.022 & 0.038 \\
$2011-2012$ & & & & & 0.006 & 0.024 \\
& 38654 & $5 \mathrm{~min}$ & 0.380 & 0.121 & 0.0115 & 0.022 \\
& 12550 & $15 \mathrm{~min}$ & 0.411 & 0.115 & 0.003 & 0.027 \\
& 6024 & $30 \mathrm{~min}$ & 0.422 & 0.119 & -0.019 & 0.033 \\
\hline
\end{tabular}

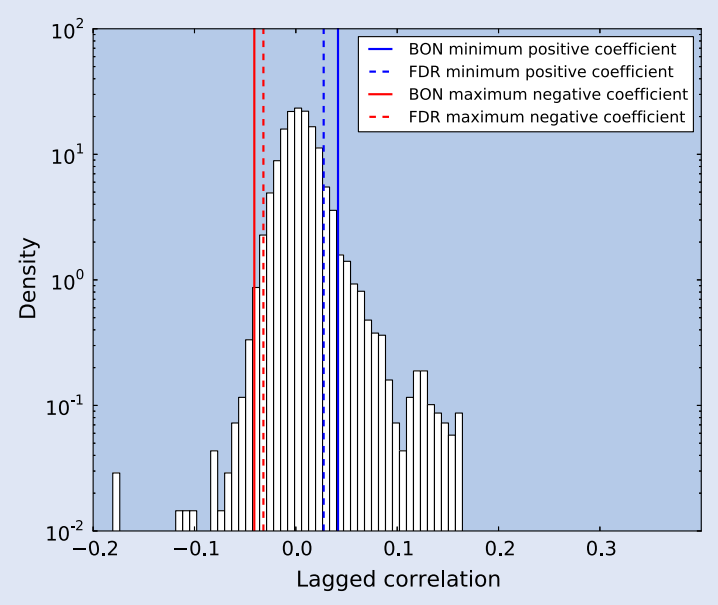

Figure 2. Distribution of lagged correlation coefficients for all $N=100$ stocks at a time horizon $h=15 \mathrm{~min}$. The minimum positive coefficients and maximum negative coefficients selected using both Bonferroni and FDR filtering procedures are shown. We note that these methods select coefficients from the tails of the distribution, without fixing a uniform threshold for all pairs of stocks.

and standard deviation $\sigma_{C}$ of entries in the lagged correlation matrix $C$.

Figure 2 displays bounds on the positive and negative coefficients selected by this method for both Bonferroni and FDR networks at a time horizon of $h=15 \mathrm{~min}$.

In figure 3 , we display plots of each statistically validated lagged correlation network obtained from the 2011-2012 data (Bonferroni correction). At time horizons of $h=130$ and $h=65 \mathrm{~min}$ we validate one and three links, respectively. It is somewhat remarkable that we uncover any persistent relationships at such long time horizons.

We see a striking increase in the number of validated links at small intraday time horizons, below $h=30 \mathrm{~min}$ in particular. This is likely due to a confluence of two effects: (i) with decreasing $h$, we increase the length $T$ of our time series, gaining statistical power and therefore the ability to reject the null hypothesis; (ii) at small $h$, we approach the timescales over which information and returns spill over across different equities. In appendix 3, we provide evidence that diminishing the time horizon $h$ reveals more information about the system than is obtained by increasing the time series length $T$ alone.

It is clear visually that the validated links of positive correlation vastly outnumber the validated links of negative correlation. We plot the number of validated links in both the Bonferroni and FDR networks for the 2002-2003 and 20112012 data-sets in figure 4, where the decrease in number of all validated links for increasing time horizon is apparent. Note that for a given time horizon, we usually validate more links in the 2002-2003 data-set than in the 2011-2012 data-set. This suggests that there has been an increase in market efficiency over the past decade. We revisit this idea in subsequent portions of this paper, where we study the properties of the network inand out-degree distributions and the characterization of threenode motifs.

We also explore how the number of validated links decreases for a fixed time horizon $h$, but a changing time lag. We build a lag $l$ into the lagged correlation matrix (2) by excluding the last $l$ returns of each trading day from matrix $A$ and the first $l$ returns of each trading day from matrix $B$. Thus, the present analysis uses $l=1$. In appendix 3, we plot the decreasing number of validated links with increasing $l$ for $h=15 \mathrm{~min}$. 


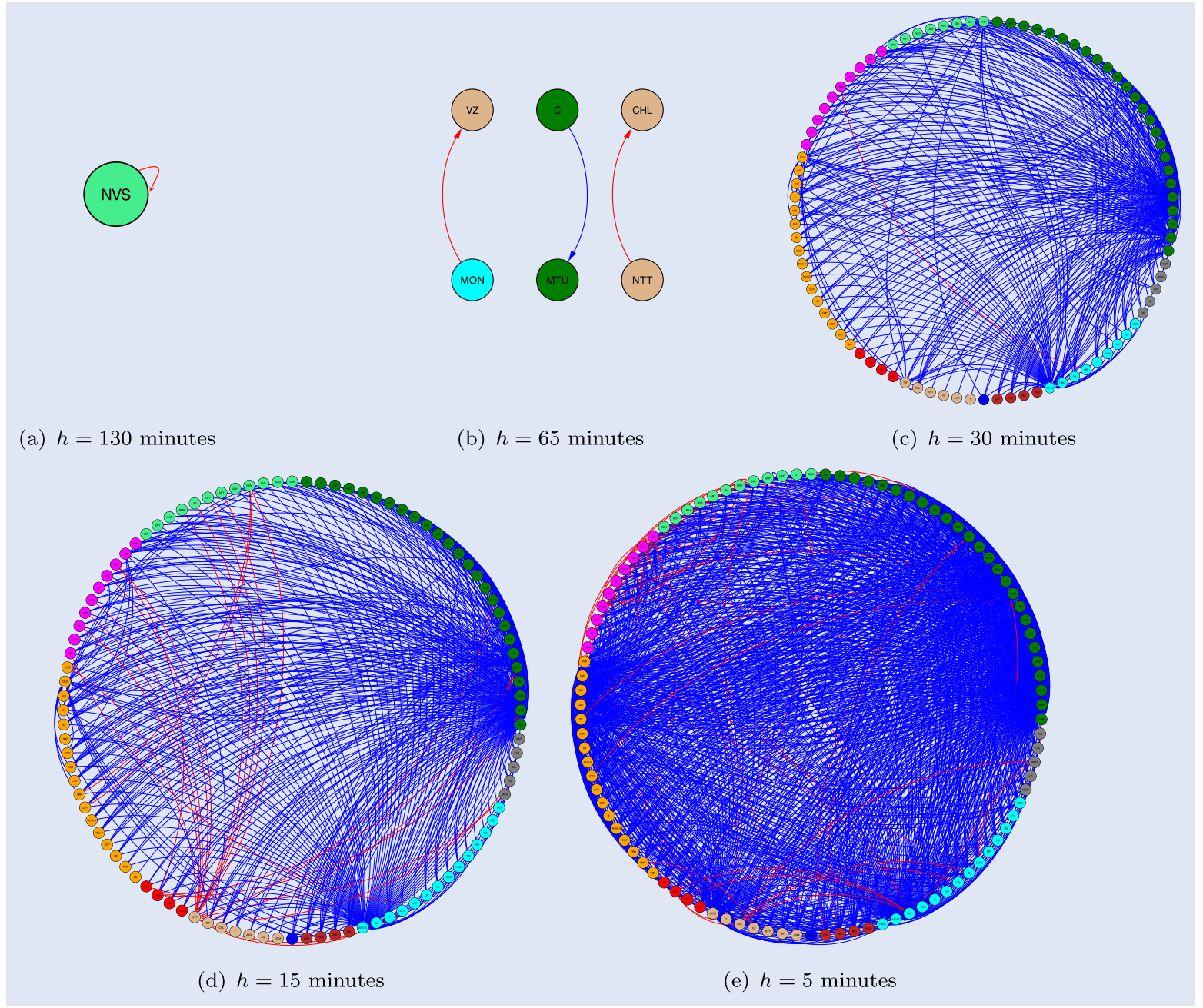

Figure 3. Illustrations of Bonferroni networks constructed from statistically-validated lagged correlations for various time horizons $h$. Data were obtained from returns of large market-capitalization companies on the NYSE in 2011-2012. Nodes are coloured by industry. Blue links represent positive correlations; red links represent negative correlations.

We must also measure the extent to which the number of validated lead-lag relationships can be disentangled from the strength of those relationships. Figure 5 thus shows plots of the average magnitude of lagged correlation coefficients selected by the Bonferroni and FDR networks. Although we validate more links at small time horizons, we note that the average magnitude of the selected coefficients tends to decrease. At short time horizons $h$ we correlate time series of comparatively large length $T$, narrowing the distribution of entries in the shuffled lagged correlation matrix $\widetilde{C}$ and gaining statistical power. We are thus able to reject the null hypothesis even for lead-lag relationships with a modest correlation coefficient.

Finally, in figure 6, we characterize the topologies of the statistically validated networks by studying the properties of their in-degree and out-degree distributions. We make two observations. First, we note that both the in-degree and outdegree distributions appear more homogeneous in the 20022003 period than the 2011-2012 period, i.e. the 2011-2012 data exhibit large heterogeneities, particularly in the in-degree distributions, in which many nodes have small degrees but few nodes have very large degrees, as can be seen in the extended tails of the distributions. Second, we observe that in both the 2002-2003 and 2011-2012 data, there are more nodes with large in-degrees than out-degrees. Although few individual stocks have a strong influence on the larger financial market, it appears that the larger financial market has a strong influence on many individual stocks, especially at short time horizons.

We further investigate this point by studying the relative occurrence of three-node network motifs in the Bonferroni networks (Milo et al. 2002). We find that, of all motifs featuring more than one link, the '021U' motif (two nodes influencing a common third node) occurs frequently in the recent data, and in fact occurs in over $80 \%$ of node triplets having more than one link between them for time horizons greater than $h=65 \mathrm{~min}$. In the 2002-2003 data, this motif is also the most common at every time horizon except $h=65 \mathrm{~min}$. Figure 7 plots the occurrence frequencies of these motifs. These features can be related to the information efficiency of the market. In the 2011-2012 data-set, we find a dominant motif in which a large number of stocks influence only a few other stocks. Predictive information 


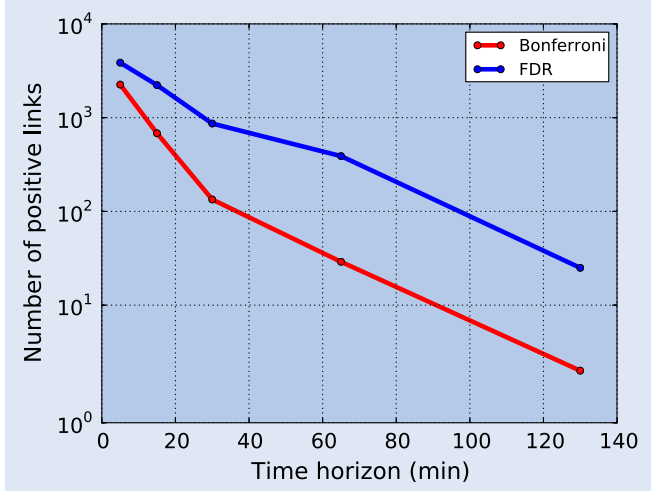

(a) Links of positive correlation, 2002-2003

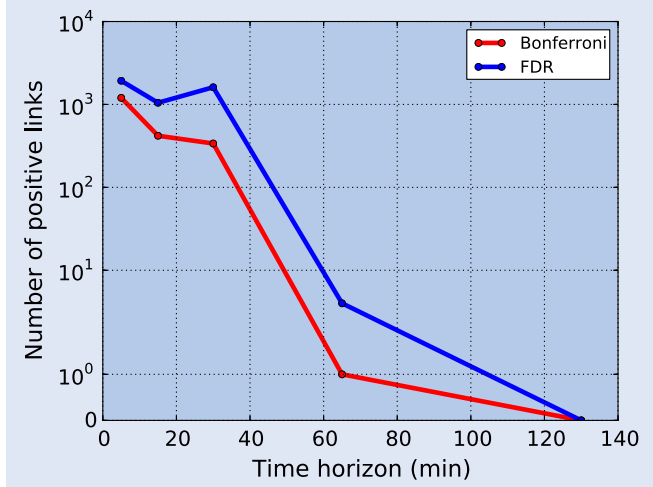

(c) Links of positive correlation, 2011-2012

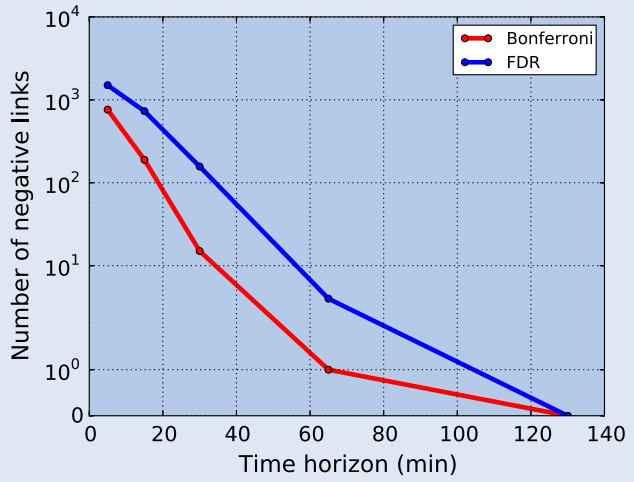

(b) Links of negative correlation, 2002-2003

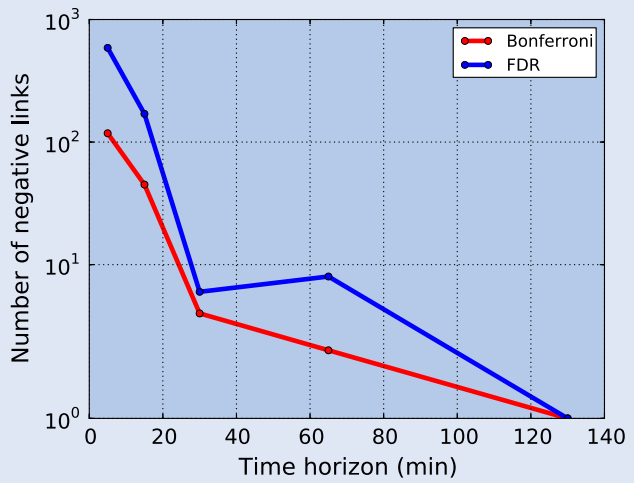

(d) Links of negative correlation, 2011-2012

Figure 4. Plots of the number of positive and negative validated links for both Bonferroni and FDR lagged correlation networks. The decrease in number of validated links for increasing time horizon is apparent in both the 2002-2003 and 2011-2012 data-sets. The vertical axis is presented on a logarithmic scale that is linearized near zero.

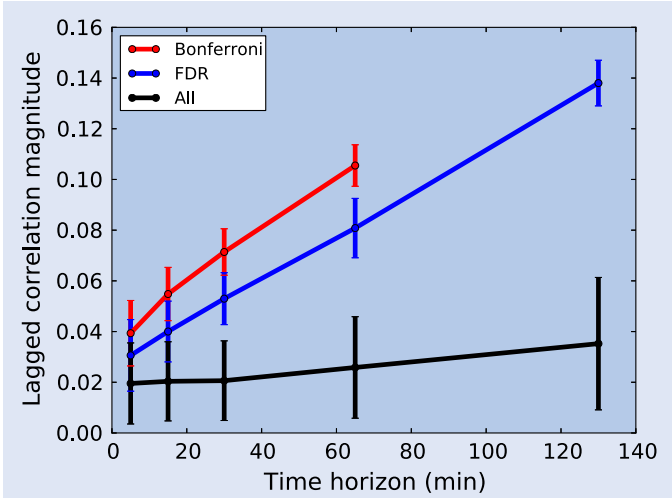

(a) $2002-2003$

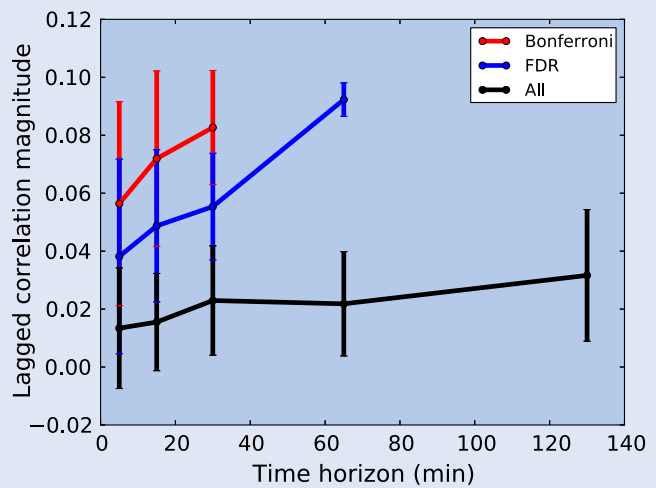

(b) 2011-2012

Figure 5. Average magnitude (absolute value) of lagged correlation coefficients filtered in Bonferroni and FDR networks. Magnitudes appear to grow with increasing time horizon of return sampling. Error bars represent plus-or-minus one standard deviation. Results are displayed only for networks containing at least five links.

regarding a given stock, therefore, tends to be encoded in the price movements of many other stocks and so is difficult to extract and exploit. In contrast, the distributions of degrees and motifs in the 2002-2003 data are more homogeneous. Although there are more nodes with large in-degrees, there are also more nodes with large out-degrees. If a stock has a large out-degree, its price movements influence the price movements of many other stocks. These sources of exploitable information have all but disappeared over the past decade.

\section{Synchronous correlation networks}

To construct synchronous correlation networks using the methodology described in section 2 , we use the unfiltered 


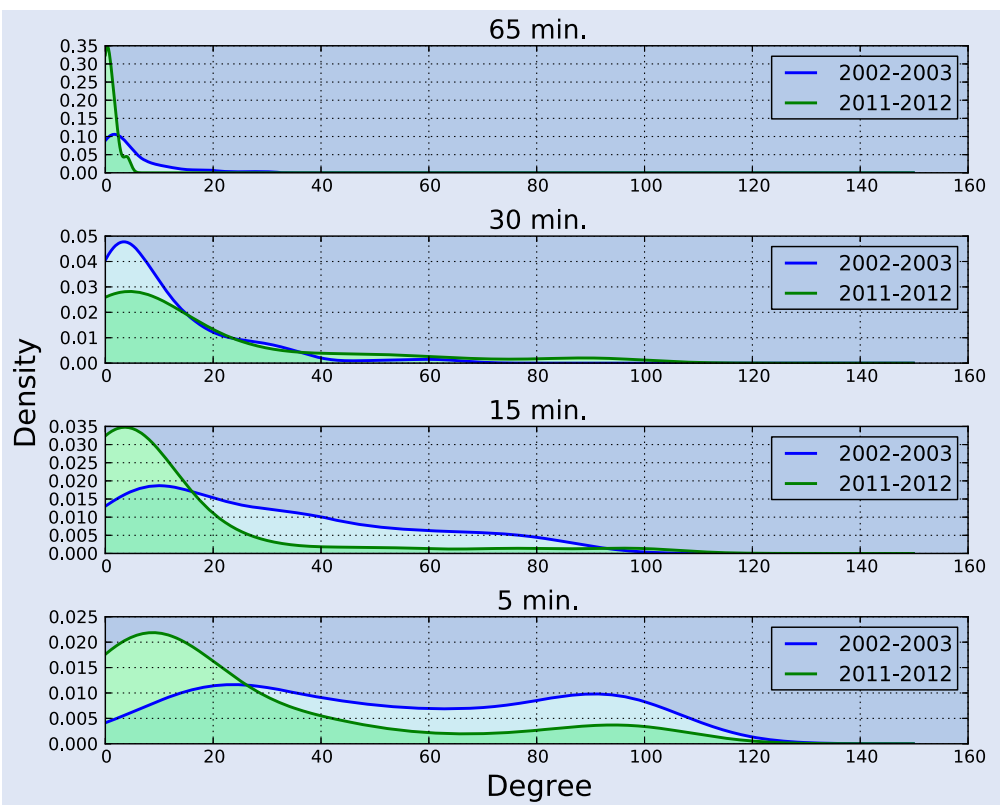

(a) In-degree distributions of FDR networks
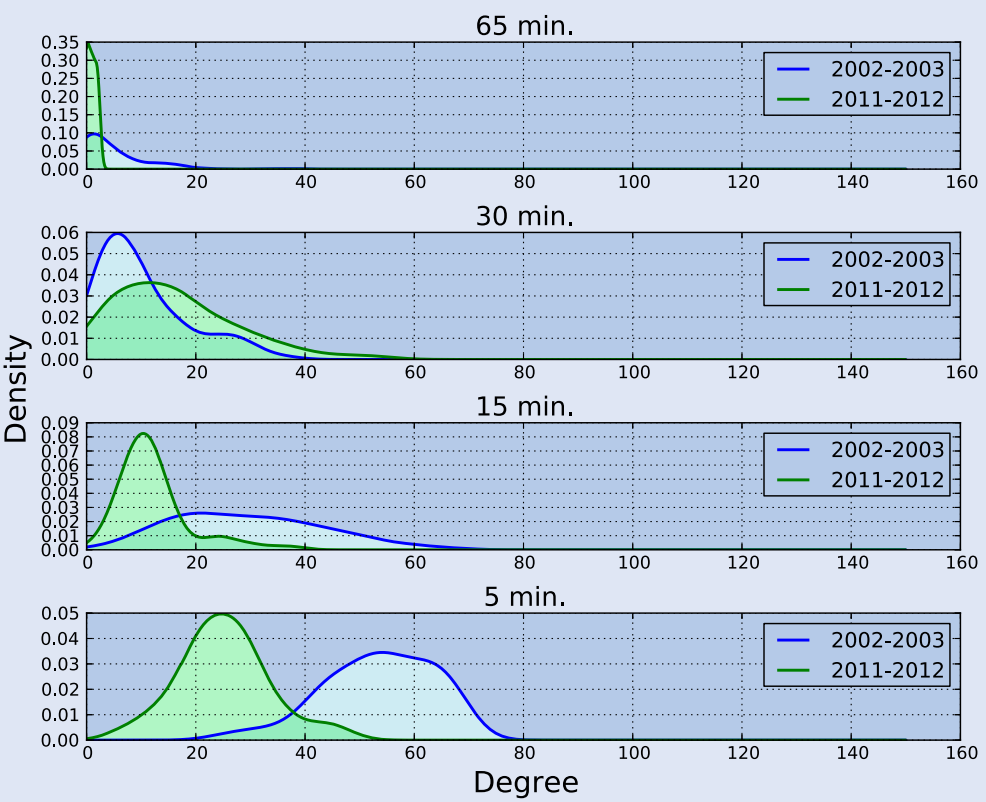

(b) Out-degree distributions of FDR networks

Figure 6. In- and out-degree distributions for FDR networks from 2002-2003 (blue) and 2011-2012 (green). Smoothed distributions are obtained using a kernel density estimate with a Gaussian kernel. With the exception of the $h=30$ min in-degree distributions, at each of the 30,15, and 5 min time horizons the distributions from 2002-2003 and 2011-2012 are statistically distinct $(p<0.05$, all test statistics $W>130$, two-tailed two-sample Wilcoxon rank-sum tests, Bonferroni correction applied).

columns of $R$ as our time series such that each entry $C_{m, n}$ of the empirical correlation matrix is the Pearson correlation between columns $m$ and $n$ of $R$. We then independently shuffle the columns of $R$, without replacement, when constructing the surrogated time series. We find that with the same significance threshold of $p=0.01$, in 2011-2012 both the Bonferroni and FDR networks are almost fully connected, with well over 4500 of the $N(N-1) / 2=4950$ possible links validated in all networks over all time horizons. Our method is thus quite sensitive to the presence or absence of correlations between time series.

Figure 8 plots the empirical synchronous correlations against time horizon for all stocks considered in both data-sets. We see a clear increase in the magnitude of these coefficients as the time horizon grows, a phenomenon known as the Epps Effect (Epps 1979, Tumminello, Di Matteo et al. 2007). It is known 

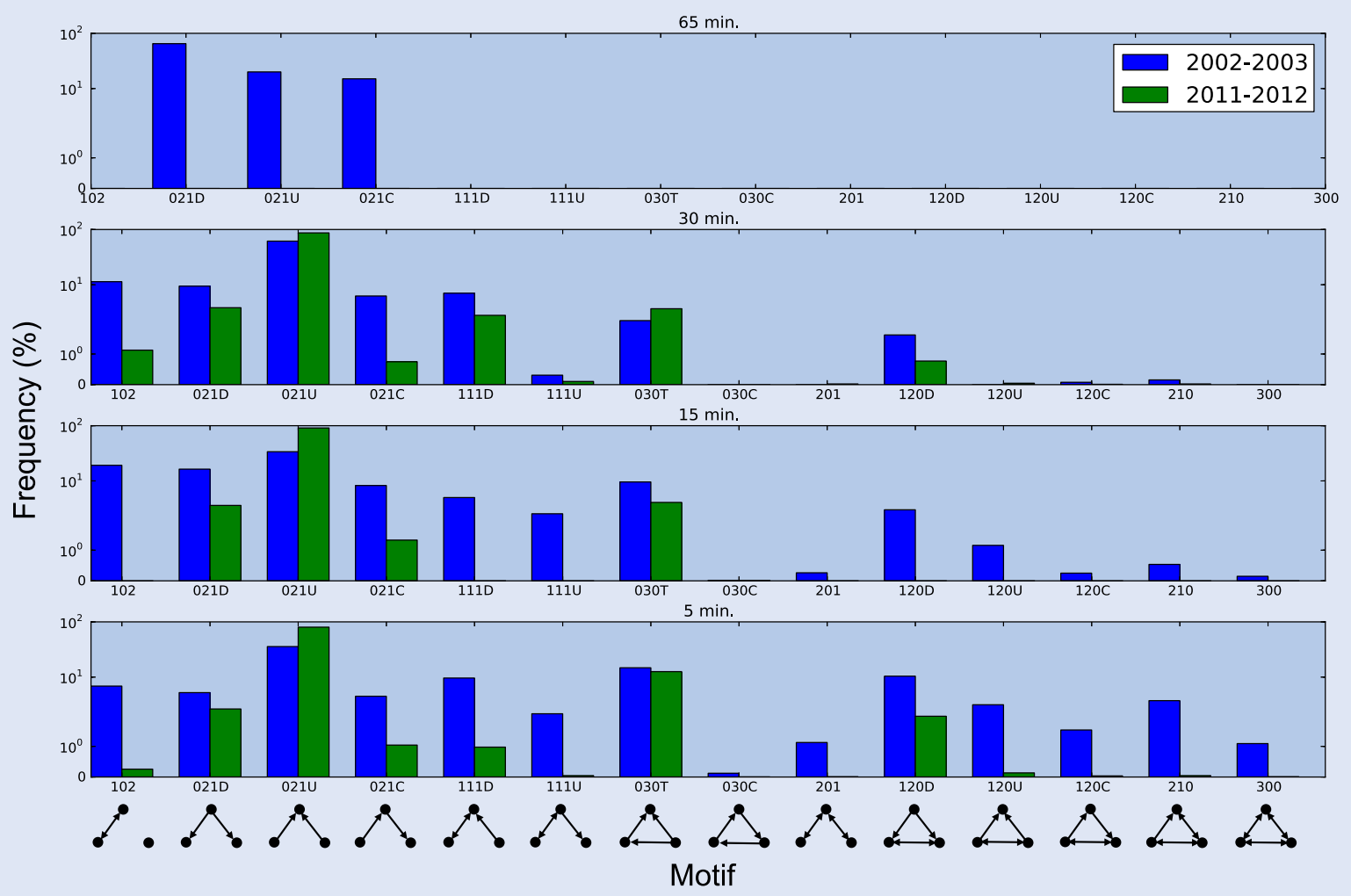

Figure 7. Percentage occurrence of all 14 possible directed three-node motifs with more than one link in Bonferroni networks. The total number of such motifs in 2002-2003 are $40(h=65 \mathrm{~min}), 1296(h=30 \mathrm{~min}), 17545(h=15 \mathrm{~min})$ and $92673(h=5 \mathrm{~min})$. In 2011-2012, these counts are $0(h=65 \mathrm{~min}), 8,710(h=30 \mathrm{~min}), 13850(h=15 \mathrm{~min})$ and $46687(h=5 \mathrm{~min})$.

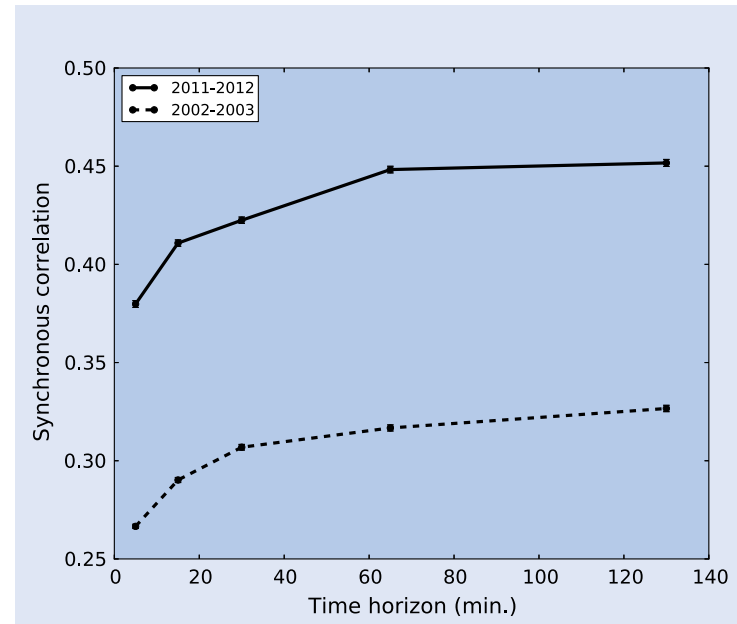

(a) Epps curves for 2002-2003 and 2011-2012 data.

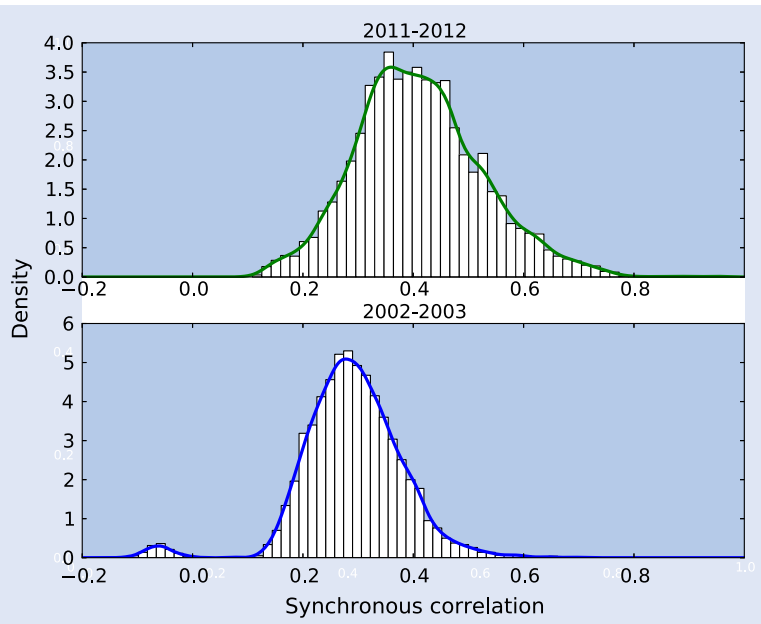

(b) Distributions of correlations at a 15 minute time horizon

Figure 8. (a) Plot of mean synchronous correlation coefficients in both 2002-2003 and 2011-2012 data. Error bars represent plus-or-minus one standard deviation of the mean. (b) Histograms of correlation coefficients for returns sampled at a 15 min time horizon. Solid curves show kernel density estimates using a Gaussian kernel. Depicted distributions are statistically distinct $(p<0.001$, test statistic $W=19415612$, two-tailed two-sample Wilcoxon rank-sum test).

that lagged correlations may in part contribute to this effect (Toth and Kertesz 2009). The extent of this contribution is an active area of investigation (Curme et al. in preparation). The synchronous correlations are also significantly higher in the recent data, suggesting that, despite the increased efficiencies shown in figure 4 , there is also an increase in co-movements in financial markets since 2003, heightening the risk of financial contagion (see for example Song et al. 2011, Kenett, Raddant et al. 2012).

Figure 8(b) shows the distribution of correlation coefficients at $h=15$ min for both 2002-2003 and 2011-2012 data-sets. We observe a slightly bi-modal distribution of synchronous 
correlation coefficients in the 2002-2003 data across all time horizons $h$. Most coefficients are positive, but there is also a small number of negative coefficients among these high market capitalization stocks. This quality disappears in the 2011-2012 data, and all correlation coefficients are positive.

\section{Discussion}

In this paper, we propose a method for the construction of statistically validated correlation networks. The method is applicable to the construction of both lagged (directed) and synchronous (undirected) networks, and imposes no topological constraints on the networks. The sensitivity of the method to small deviations from the null hypothesis of uncorrelated returns makes it less useful for studying the synchronous correlations of stocks, as these equities tend to display a considerable degree of correlation and we validate almost all possible links in the network. The method is apt, however, for the study of lagged correlation networks. We are able to adjust the sensitivity of the method with our choice of $p$-value and protocol for multiple comparisons. Here we show that, with the conservative Bonferroni correction and $p$-value $=0.01$, we are able to compare changes in network connectivity with increasing return sampling frequency between old and new data-sets. The primary drawback to our method is its computational burden, which grows as $\mathcal{O}\left(N^{4}\right)$ for $N$ time series.

We find that for timescales longer than one hour, significant lead-lag relationships that capture return and information spillover virtually disappear. For timescales smaller than $30 \mathrm{~min}$, however, we are able to validate hundreds of relationships. According to the efficient market hypothesis, there can be no arbitrage opportunities in informationally efficient financial markets. However, lagged correlations may not be easily exploitable due to the presence of market frictions, including transaction costs, the costs of information processing and borrowing constraints.

Between the time periods 2002-2003 and 2011-2012, the synchronous correlations among these high market capitalization stocks grow considerably, but the number of validated lagged-correlation relationships diminish. We relate these two behaviours to an increase in the risks of financial contagion and an increase in the informational efficiency of the market, respectively. We find that networks from both periods exhibit asymmetries between their in-degree and out-degree distributions. In both there are more nodes with large in-degrees than large out-degrees, but in the 2011-2012 data, nodes with large in-degrees are represented by the extended tails of the degree distribution and, in contrast, the 2002-2003 distribution exhibits a greater uniformity. A comparison between in-degree and out-degree distributions shows that nodes with high indegree are much more likely than nodes with high out-degree, especially for the 2011-2012 data. This evidence is also interpreted in terms of informational efficiency of the market. Indeed, a large out-degree of a stock implies that knowledge of its return, at a given time, may provide information about the future return of a large number of other stocks. On the other hand, a large in-degree of a stock indicates that information about its return at a given time can be accessed through the knowledge of past returns of many stocks. There are also many more nodes with large out-degrees in the 2002-2003 data than in the 2011-2012 data. We relate these observations to an increased information efficiency in the market. Such an interpretation is also supported by the analysis of three-node motifs, which shows an apparent dominance of motif $021 \mathrm{U}$ with respect to all the others.

In the future, we could extend this work by incorporating a prediction model to measure the degree to which the information contained in these validated networks is exploitable in the presence of market frictions. We could also investigate the characteristics of nodes belonging to different industries, as well as the presence of intraday seasonalities (Allez and Bouchaud 2011, Curme et al. in preparation). Such features are potentially relevant to prediction models. Finally, although our analysis restricts itself to using the Pearson product-moment correlation, other measures, such as a lagged Hayashi-Yoshida estimator (Hayashi and Yoshida 2005), could be used to probe correlations at the smallest (inter-trade) timescales while minimizing the problem of asynchronous trades.

\section{Acknowledgements}

We thank Viktoria Dalko for useful conversations and insights, and her help with the data.

\section{Disclosure statement}

No potential conflict of interest was reported by the authors.

\section{Funding}

CC, DYK and HES wish to thank ONR [grant number N0001409-1-0380], [grant number N00014-12-1-0548], DTRA [grant number HDTRA-1-10-1-0014], [grant number HDTRA-1-091-0035], and NSF [grant number CMMI 1125290]. M.T. and R.N.M. acknowledge support from the INET research project NetHet 'New Tools in Credit Network Modeling with Heterogenous Agents'. R. N. M. acknowledge support from the FP7 research project CRISIS 'Complexity Research Initiative for Systemic InstabilitieS'.

\section{Additional Information}

The authors declare no competing financial interests.

\section{References}

Allez, R. and Bouchaud, J.P., Individual and collective stock dynamics: Intra-day seasonalities. New J. Phys., 2011, 13, 025010.

Aste, T., Shaw, W. and Di Matteo, T., Correlation structure and dynamics in volatile markets. New J. Phys., 2010, 12, 085009.

Benjamini, Y. and Hochberg, Y., Controlling the false discovery rate: A practical and powerful approach to multiple testing. J. Roy. Stat. Soc. Ser. B (Methodological), 1995, 289-300.

Billio, M., Getmansky, M., Lo, A. and Pelizzon, L., Econometric measures of connectedness and systemic risk in the finance and insurance sectors. J. Financ. Econ., 2012, 104, 535-559. 
Biroli, G., Bouchaud, J.P. and Potters, M., The student ensemble of correlation matrices: Eigenvalue spectrum and Kullback-Leibler entropy. Acta Phys. Pol. B, 2007, 38, 4009-4026.

Bonanno, G., Caldarelli, G., Lillo, F. and Mantegna, R.N., Topology of correlation-based minimal spanning trees in real and model markets. Phys. Rev. E, 2003, 68, 046130.

Bonanno, G., Lillo, F. and Mantegna, R.N., High-frequency crosscorrelation in a set of stocks. Quant. Finance, 2001, 1, 96-104.

Borghesi, C., Marsili, M. and Miccichè, S., Emergence of timehorizon invariant correlation structure in financial returns by subtraction of the market mode. Phys. Rev. E, 2007, 76, 026104.

Campbell, R., Forbes, C., Koedijk, K. and Kofman, P., Increasing correlations or just fat tails? J. Emp. Finance, 2008, 15, 287-309.

Carbone, A., Detrending moving average algorithm: A brief review. Proceedings of the Science and Technology for Humanity (TICSTH), 2009 IEEE Toronto International Conference, Toronto, ON, pp. 691-696, 2009.

Cecchetti, S. and Kharroubi, E., Reassessing the impact of finance on growth. BIS Working Paper, 2012. Available online at SSRN: http://ssrn.com/abstract=2117753.

Cizeau, P., Potters, M. and Bouchaud, J., Correlation structure of extreme stock returns. Quant. Finance, 2001, 1, 217-222.

Curme, C., Tumminello, M., Mantegna, R.N., Stanley, H.E. and Kenett, D.Y., How lead-lag correlations affect the intra-day pattern of collective stock dynamics. Manuscript in preparation.

De Jong, F., Nijman, T. and Röell, A., Price effects of trading and components of the bid-ask spread on the Paris Bourse. J. Emp. Finance, 1996, 3, 193-213.

Efron, B. and Tibshirani, R., An Introduction to the Bootstrap, Vol. 57, 1993 (CRC Press: Boca Raton, FL).

Epps, T., Comovements in stock prices in the very short run. J. Am. Stat. Assoc., 1979, 291-298.

Forbes, K. and Rigobon, R., No contagion, only interdependence: Measuring stock market comovements. J. Finance, 2002, 57, 2223-2261.

Gopikrishnan, P., Plerou, V., Liu, Y., Amaral, L., Gabaix, X. and Stanley, H., Scaling and correlation in financial time series. Phys. A: Stat. Mech. Appl., 2000, 287, 362-373.

Gopikrishnan, P., Rosenow, B., Plerou, V. and Stanley, H., Quantifying and interpreting collective behavior in financial markets. Phys. Rev. E, 2001, 64, 035106

Hall, R.E., Why does the economy fall to pieces after a financial crisis? J. Econ. Persp., 2010, 24, 3-20.

Havlin, S., Kenett, D.Y., Ben-Jacob, E., Bunde, A., Cohen, R., Hermann, H., Kantelhardt, J., Kertész, J., Kirkpatrick, S., Kurths, J., Portugali, J. and Solomon, S., Challenges in network science: Applications to infrastructures, climate, social systems and economics. Eur. Phys. J.-Special Topics, 2012, 214, 273-293.

Hayashi, T. and Yoshida, N., On covariance estimation of nonsynchronously observed diffusion processes. Bernoulli, 2005, 11, 359-379.

Huth, N. and Abergel, F., High frequency lead/lag relationshipsEmpirical facts. J. Emp. Finance, 2014, 26, 41-58.

Kenett, D.Y., Preis, T., Gur-Gershgoren, G. and Ben-Jacob, E., Quantifying meta-correlations in financial markets. Europhys. Lett., 2012, 99, 38001.

Kenett, D.Y., Raddant, M., Lux, T., Ben-Jacob, E., Evolvement of uniformity and volatility in the stressed global financial village. PloS One, 2012, 7, e31144.

Kenett, D.Y., Tumminello, M., Madi, A., Gur-Gershgoren, G., Mantegna, R.N. and Ben-Jacob, E., Dominating clasp of the financial sector revealed by partial correlation analysis of the stock market. PloS One, 2010, 5, e15032.

Kenney, J.F. and Keeping, E.S., Mathematics of Statistics, Part 2, 2nd ed, 1962 (D. Van Nostrand Company Inc.: Princeton, NJ).

Laloux, L., Cizeau, P., Potters, M. and Bouchaud, J., Random matrix theory and financial correlations. Int. J. Theor. Appl. Finance, 2000, 3, 391-398

Lo, A.W. and MacKinlay, A.C., Stock market prices do not follow random walks: Evidence from a simple specification test. Rev. Financ. Stud., 1988, 1, 41-66.
Malkiel, B.G., The efficient market hypothesis and its critics. J. Econ. Persp., 2003, 17, 59-82.

Malkiel, B.G. and Fama, E.F., Efficient capital markets: A review of theory and empirical work. J. Finance, 1970, 25, 383-417.

Mantegna, R.N., Hierarchical structure in financial markets. Eur. Phys. J. B-Cond. Matter Comp. Syst., 1999, 11, 193-197.

Milo, R., Shen-Orr, S., Itzkovitz, S., Kashtan, N., Chklovskii, D. and Alon, U., Network motifs: Simple building blocks of complex networks. Science, 2002, 298, 824-827.

Munnix, M., Schafer, R. and Guhr, T., Impact of the tick-size on financial returns and correlations. Phys. A: Stat. Mech. Appl., 2010, 389, 4828-4843.

Onnela, J., Chakraborti, A., Kaski, K. and Kertesz, J., Dynamic asset trees and Black Monday. Phys. A: Stat. Mech. Appl., 2003, 324, 247-252.

Podobnik, B. and Stanley, H.E., Detrended cross-correlation analysis: A new method for analyzing two nonstationary time series. Phys. Rev. Lett., 2008, 100, 084102-1-084102-4.

Pollet, J. and Wilson, M., Average correlation and stock market returns. J. Financ. Econ., 2010, 96, 364-380.

Shmilovici, A., Alon-Brimer, Y. and Hauser, S., Using a stochastic complexity measure to check the efficient market hypothesis. Comput. Econ., 2003, 22, 273-284.

Song, D.M., Tumminello, M., Zhou, W.X. and Mantegna, R.N., Evolution of worldwide stock markets, correlation structure, and correlation-based graphs. Phys. Rev. E, 2011, 84, 026108.

Tobin, J., A general equilibrium approach to monetary theory. $J$. Money Credit Bank., 1969, 1, 15-29.

Toth, B. and Kertesz, J., The Epps effect revisited. Quant. Finance, 2009, 9, 793-802.

Tumminello, M., Aste, T., Di Matteo, T. and Mantegna, R.N., A tool for filtering information in complex systems. Proc. Nat. Acad. Sci. USA, 2005, 102, 10421-10426.

Tumminello, M., Coronnello, C., Lillo, F., Miccichè, S. and Mantegna, R.N., Spanning trees and bootstrap reliability estimation in correlation based networks. Int. J. Bifurcat. Chaos, 2007, 17, 2319-2329.

Tumminello, M., Di Matteo, T., Aste, T. and Mantegna,, R.N., Correlation based networks of equity returns sampled at different time horizons. Eur. Phys. J. B-Cond. Matter Comp. Syst., 2007, 55, 209-217.

Tumminello, M., Lillo, F. and Mantegna, R.N., Correlation, hierarchies, and networks in financial markets. J. Econ. Behav. Org., 2010, 75, 40-58.

Tumminello, M., Miccichè, S., Lillo, F., Piilo, J. and Mantegna, R.N., Statistically validated networks in bipartite complex systems. PloS One, 2011, 6, e17994.

\section{Appendix 1. Probability of a false positive link}

The one-tailed $p$-value associated with positive correlations represents the probability of observing a correlation between two elements, $i$ and $j$, that is larger than or equal to the one observed, $\rho_{o b s}$, under the null hypothesis that $i$ and $j$ are uncorrelated,

$$
p \text {-value }\left(\rho_{o b s}\right)=P\left(\rho>\rho_{\text {obs }}\right) .
$$

Our objective in the paper is to select all the correlations with a $p$-value smaller than a given univariate statistical threshold $q_{0}$, e.g. $q_{0}=0.01$ or $q_{0}=0.05$, corrected for multiple hypothesis testing through the Bonferroni correction, that is, divided by the total number of tests, $N^{2}$ in our case (where $N=100$ is the number of stocks). The question is: what is the probability that a correlation with a $p$-value $p$ larger or equal to $p_{0}=q_{0} / N^{2}$ is (wrongly) indicated as a statistically significant one according to the shuffling method? Operatively, what is the probability that, over the $Q=k N^{2}$ independent replicates of the data, a correlation between $i$ and $j$ larger than the observed one has never been observed?

If we set the $p$-value, $p$, of $\rho_{\text {obs }}$ equal to $\frac{q}{N^{2}}$ (where $q$ is a quantity that ranges between 0 and $N^{2}$ ), the question is: what is the probability that, over $Q=k N^{2}$ independent draws $\left(Q=100 \cdot N^{2}=10^{6}\right.$ 
bootstrap replicates with our method) a value of correlation larger than $\rho_{\text {obs }}$ is never obtained? This probability is

$$
P(\operatorname{null} \mid p)=(1-p)^{Q},
$$

where 'null' indicates the event that a value of correlation larger than $\rho_{o b s}$ has never been obtained over $Q=k N^{2}$ random replicates of data. This probability can be used to calculate the probability that $p=q / N^{2}$ is larger than or equal to $p_{0}=q_{0} / N^{2}$, conditioned to the event that a value of correlation larger than $\rho_{o b s}$ has never been obtained over $Q=k N^{2}$ draws. This is done using Bayes' rule, under the assumption that the marginal distribution of $p$-value $p$ is uniform in $[0,1]$, i.e. the density function is $f(p)=1$ and then, integrating over $p$,

$$
\begin{aligned}
P( & \left.\geq p_{0} \mid \text { null }\right)=\int_{p_{0}}^{1} \frac{P(\operatorname{null} \mid p) f(p)}{P(\text { null })} \mathrm{d} p \\
& =\int_{p_{0}}^{1}(Q+1)(1-p)^{Q} \mathrm{~d} p=\left(1-p_{0}\right)^{Q+1},
\end{aligned}
$$

where we used the fact that $P($ null $)=\int_{0}^{1} P(\operatorname{null} \mid p) f(p) \mathrm{d} p=\frac{1}{Q+1}$. In our method, $k=100$, and the sample size is $N=100$. Therefore,

$P\left(p \geq p_{0} \mid\right.$ null $)=\left(1-\frac{q_{0}}{N^{2}}\right)^{k N^{2}+1} \cong\left(1-\frac{q_{0}}{N^{2}}\right)^{k N^{2}} \cong e^{-k q_{0}}$.

(A4)

It is interesting to note that, as soon as the level of statistical significance is corrected through the Bonferroni correction $\left(p_{0}=q_{0} / N^{2}\right)$, where $q_{0}$ is the univariate level of statistical significance, and the number, $Q$, of independent replicates is a multiple of the number of tests, $Q=k N^{2}$, the probability $P\left(p \geq p_{0} \mid\right.$ null $)$ is approximately independent of the sample size $(N)$.

With our approximated method to estimate correlation $p$-values, the probability that we select a positive correlation as a statistically significant one at the confidence level $p_{0}=q_{0} / N^{2}=0.01 / 100^{2}=10^{-6}$, while it is actually not significant at that level of statistical confidence, is $P(q \geq 0.01 \mid$ null $)=\frac{1}{e} \cong 0.368$. However, the probability that a significant correlation according to our method has a $p$-value larger then $0.05 / N^{2}=0.05 / 100^{2}=5 \times 10^{-6}$ is already quite small: $P(q \geq 0.05 \mid$ null $)=\frac{1}{e^{5}} \cong 0.0067$. In other words, if we obtain a validated network with 1000 links, i.e. 1000 validated positive correlations according to our approximated method, we expect that, on average, only seven correlations will have a one-tailed $p$-value larger than $0.05 / 100^{2}=5 \times 10^{-6}$.

\section{Appendix 2. Comparison of the bootstrap method and an analytical one to calculate correlation p-values}

Here we compare (for a sub-set of our data) the number of significant correlations obtained according to the presented bootstrap approach and the number of significant correlations that we may have obtained relying upon the analytical distribution of sample pair correlations of normally distributed data.

If $x$ and $y$ are uncorrelated variables that follow a normal distribution, then the probability density function of the sample correlation coefficient, $r$, between $x$ and $y$ is Kenney and Keeping (1962)

$$
f(r, T)=\frac{\left(1-r^{2}\right)^{\frac{T-1}{2}-2}}{B\left(\frac{1}{2}, \frac{T-1}{2}-1\right)},
$$

where $T$ is the length of the sample and $B(q, p)$ is the Euler beta function of parameters $q$ and $p$. Given a level of statistical significance, $q_{0} / N^{2}$ (already corrected for multiple hypothesis testing), $f(r, T)$ can be used to set a threshold for the correlation value $\rho_{t}$ such that the probability $P\left(\rho>\rho_{t}\right)=\frac{q_{0}}{N^{2}}$ is

$$
P\left(\rho>\rho_{t}\right)=\int_{\rho_{t}}^{1} f(r, T) \mathrm{d} r=\frac{q_{0}}{N^{2}} .
$$

According to this analysis, for a data sample of $N$ time series, each one of length $T$, we can say that an observed correlation, $\rho_{o b s}$, is statistically significant if $\rho_{o b s}>\rho_{t}$, where $\rho_{t}$ is obtained by (numerically) solving the previous non linear equation.

Table B1 shows the 2002-2003 data-set and reports the length of data series used to calculate lagged correlations (column 1) at a given time horizon (column 2), the quantity $\rho_{t}$ such that $P\left(\rho>\rho_{t}\right)=$ $0.01 / N^{2}=10^{-6}$ (column 3 ), the number of validated positive correlations (column 4), and the number of validated negative correlations (column 5)

Table B2 shows the number of validated positive correlations (i) according to the shuffling method (column 3), (ii) according to the analytical method discussed above (column 4) and (iii) common to both methods (column 5). The results reported in the table show that the bootstrap method we used is more conservative than the analytical method based on the assumption that return time series follow a normal distribution. Indeed, the number of validated positive correlations according to the bootstrap method is always smaller than the one obtained using the theoretical approach. Furthermore, most of the correlations validated according to the bootstrap method are also validated according to the theoretical method.

A similar discussion can be held about the validation of negative correlations.

\section{Appendix 3. Effect of lag and time series length on vali- dated links for a fixed time horizon}

We explore how the number of validated links decreases when the time horizon $h$ is fixed and the time lag variable $l$ increases. A lag $l$ is built into the lagged correlation matrix (2) by excluding the last $l$ returns of each trading day from matrix $A$ and the first $l$ returns of each trading day from matrix $B$. Thus, the results presented in the main text are restricted to $l=1$. Figure $\mathrm{C} 1$ plots the number of positive links and negative links validated in the 2011-2012 data for $h=15 \mathrm{~min}$ as $l$ increases. Although for this $h$ the length $T$ of the time series in $A$ and $B$ decrease by only $\approx 4 \%$ for each additional lag $l$ (as each $390 \mathrm{~min}$ trading day includes $390 / 15-l=26-l$ returns), we observe a sharp decrease in the number of validated links as $l$ increases. The number of validated negative links is an order of magnitude smaller than the number of positive links, so the small peak in negative links at $l=3$ for the FDR network is likely an artifact of noise.

We also investigate the effect of the time series length $T$ on the numbers of validated links. For $h=15 \mathrm{~min}$, we partition the entire 2011-2012 time series into segments of length $T=1004$, as this is the length of the time series for the longest time horizon considered $(h=130 \mathrm{~min})$. For each segment, we generate the lagged correlation network using $10^{6}$ surrogate time series, as before. We find that the union of all such Bonferroni networks consists of 125 distinct links, 106 of which are positive and 19 of which are negative. Although this number is $30 \%$ of the number of links validated in the $h=15 \mathrm{~min}$ network that was not partitioned $(T=12550)$, it stands in contrast to the single link that was validated in the $h=130 \mathrm{~min}$ Bonferroni network using the entire time period. The number validated in each partition is shown in figure $\mathrm{C} 2$. We can thus safely conclude that decreasing the time horizon $h$ Provides information independent of the increased time series length $T$. 
Table B1. Threshold correlation values and validated links according to a normal distribution of returns.

\begin{tabular}{lccrr}
\hline$T$ & $h$ & $\rho_{t}$ & \# pos. valid. & \# neg. valid \\
\hline 38577 & $5 \mathrm{~min}$ & 0.0242 & 2398 & 793 \\
12525 & $15 \mathrm{~min}$ & 0.0425 & 754 & 212 \\
6012 & $30 \mathrm{~min}$ & 0.0613 & 158 & 19 \\
2505 & $65 \mathrm{~min}$ & 0.0948 & 43 & 3 \\
1002 & $130 \mathrm{~min}$ & 0.1496 & 3 & 0 \\
\hline
\end{tabular}

Table B2. Comparison between number of validated links according to (1) bootstrap method and (2) a normal distribution of returns.

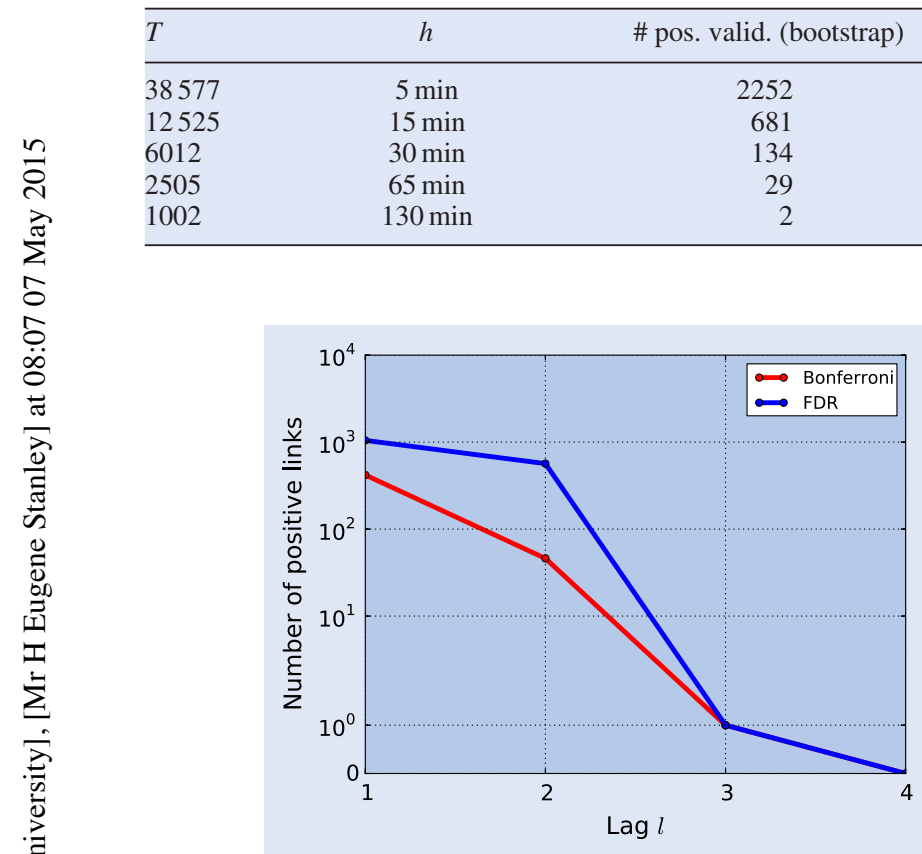

(a) Links of positive correlation

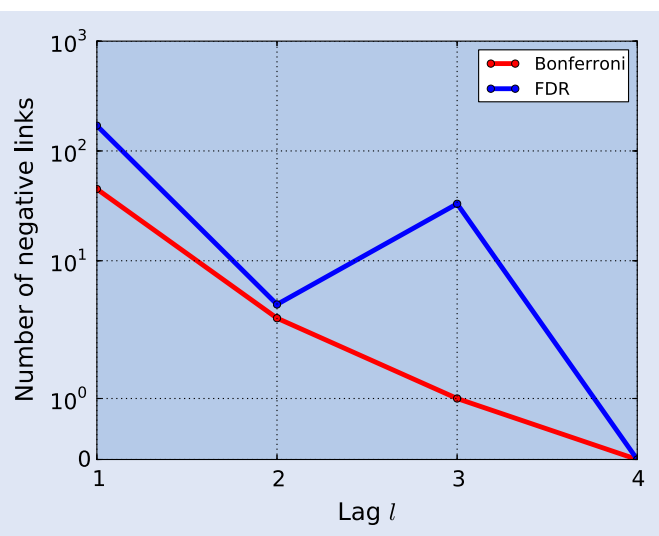

(b) Links of negative correlation

Figure C1. Numbers of positive and negative validated links for both Bonferroni and FDR correlation networks for varying lag $l$. Returns are sampled every $h=15 \mathrm{~min}$ from the 2011-2012 data.

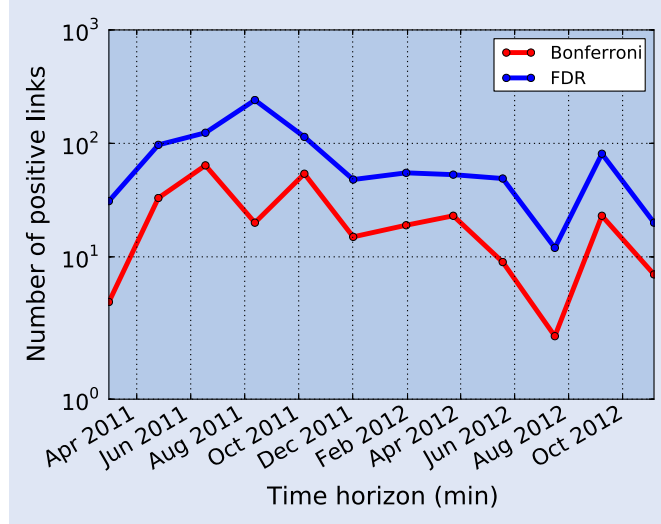

(a) Links of positive correlation

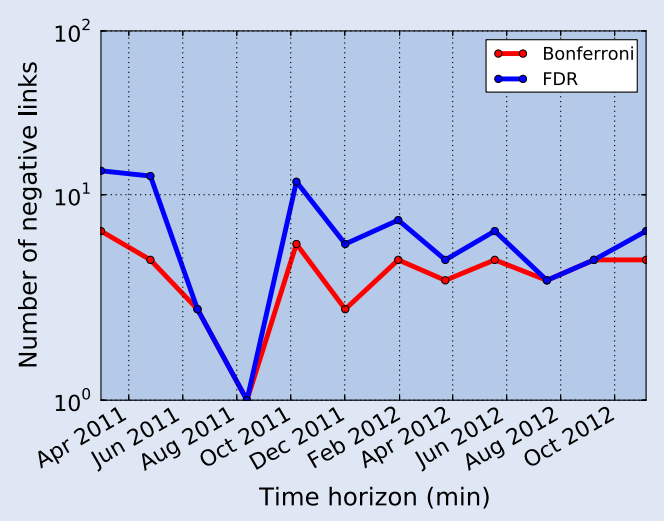

(b) Links of negative correlation

Figure C2. Numbers of positive and negative validated links for both Bonferroni and FDR lagged correlation networks for time series segments of length $T=1004$ at $h=15 \mathrm{~min}$. Horizontal axis gives date of the final return in each network. 